\title{
Oral health and plaque microbial profile in juvenile idiopathic arthritis
}

\author{
Sriharsha Grevich ${ }^{1,2^{*}}$ D, Peggy Lee ${ }^{3}$, Brian Leroux ${ }^{3}$, Sarah Ringold ${ }^{1,2,4}$, Richard Darveau ${ }^{3}$, Gretchen Henstorf ${ }^{5}$, \\ Joel Berg ${ }^{3}$, Amy Kim³ ${ }^{3}$ Elizabeth Velan ${ }^{3}$, Joseph Kelly ${ }^{3}$, Camille Baltuck ${ }^{3}$, Anne Reeves ${ }^{3}$, Hannah Leahey ${ }^{5}$, \\ Kyle Hager ${ }^{6}$, Mitchell Brittnacher ${ }^{6}$, Hillary Hayden ${ }^{6}$, Samuel Miller ${ }^{6,7,8}$, Jeffrey McLean ${ }^{3}$ and Anne Stevens ${ }^{1,2,5}$
}

\begin{abstract}
Background: The oral microbiota has been implicated in the pathogenesis of rheumatoid arthritis through activation of mucosal immunity. This study tested for associations between oral health, microbial communities and juvenile idiopathic arthritis (JIA).

Methods: A cross-sectional exploratory study of subjects aged 10-18 years with oligoarticular, extended oligoarticular and polyarticular JIA was conducted. Control groups included pediatric dental clinic patients and healthy volunteers. The primary aim was to test for an association between dental health indices and JIA; the secondary aim was to characterize the microbial profile of supragingival plaque using 165 rRNA gene sequencing.

Results: The study included 85 patients with JIA, 62 dental patients and 11 healthy child controls. JIA patients overall had significantly more gingival inflammation compared to dental patients, as evidenced by bleeding on probing of the gingiva, the most specific sign of active inflammation $(p=0.02)$. Overall, however, there was a trend towards better dental hygiene in the JIA patients compared to dental patients, based on indices for plaque, decay, and periodontitis. In the JIA patients, plaque microbiota analysis revealed bacteria belonging to genera Haemophilus or Kingella elevated, and Corynebacterium underrepresented. In poly JIA, bacteria belonging to the genus Porphyromonas was overrepresented and Prevotella was underrepresented.
\end{abstract}

Conclusion: Increased gingival inflammation in JIA was independent of general oral health, and thus cannot be attributed to poor dental hygiene secondary to disability. The variation of microbial profile in JIA patients could indicate a possible link between gingivitis and synovial inflammation.

Keywords: Juvenile idiopathic arthritis, Oral health, Gingivitis, Microbiota

\section{Background}

Juvenile idiopathic arthritis (JIA) is a common rheumatologic disorder that can lead to significant disability. The cause is not known, precluding prevention or cure. A current hypothesis is that environmental triggers interact with specific human leukocyte antigen (HLA) and innate immunity genes associated with JIA [1].

Humans have co-evolved with microorganisms that play a pivotal role in immune development and homeostasis [2]. Crosstalk between the microbiome and the

\footnotetext{
* Correspondence: Sriharsha.Grevich@seattlechildrens.org

'Seattle Children's Hospital, 4800 Sand Point Way NE, Seattle, WA 98105, USA

${ }^{2}$ Department of Pediatrics, University of Washington, 1959 NE Pacific St,

Seattle, WA 98195, USA

Full list of author information is available at the end of the article
}

immune system has been implicated in the pathogenesis of adult-onset chronic inflammatory diseases, including rheumatoid arthritis (RA) [3, 4]. Altered oral microbial communities have been found in periodontitis, and periodontitis has been associated with RA [5, 6]. Both periodontitis and RA are characterized by innate immune activation [7]. Periodontal pathogens in the subgingival plaque, some also associated with RA, have been linked to gingival proinflammatory cytokines [8]. A causal relationship is suggested by control of RA by treating periodontitis [9, 10]. Adaptive immunity has also been implicated in the mechanism, given the association of $\mathrm{P}$. gingivalis, a periodontal pathogen, with early RA, especially those patients with high levels of anticitrullinated protein antibodies ACPA [11]. 
Gene expression in JIA reflects innate and adaptive immune activation similar to that reported in periodontitis $[12,13]$. However, a mechanistic link between the two diseases is not clearly established. Whereas periodontitis is an invasive process affecting deeper structures, leading to loss of connective tissue and bone, gingivitis is inflammation in response to the bacterial biofilm on teeth, and is reversible with treatment. Although periodontitis is rare in children, gingivitis, a chronic inflammatory precursor to periodontitis, occurs in up to $70 \%$ of children 6 to 11 years old, with higher rates during adolescence [14]. Severe childhood gingivitis in children is associated with a shift in oral bacteria to pathogenic gram-negative anaerobes and spirochetes [15]. Some, but not all studies reporting higher rates of gingivitis and dental decay in children with JIA could not rule out the possibility that poor dental hygiene was due to disability from arthralgias in fingers or temporomandibular joints (TMJs) [16-19]. Evidence for P. gingivalis has been found in multiple subtypes of JIA [20].

The concept that oral pathogens may trigger or perpetuate inflammatory joint disease in RA must be tested independently in JIA. The current study aimed to distinguish signs of periodontitis or gingivitis from other oral hygiene parameters, and to begin to describe the dental microbial communities associated with JIA. We hypothesized that we would find indicators of periodontitis and distinct bacterial dysbiosis in specific JIA subtypes.

\section{Methods}

\section{Study population}

Subjects were recruited at Seattle Children's Hospital and the University of Washington/Seattle Children's Hospital Center for Pediatric Dentistry. Subjects 10-18 years were included to ensure permanent dentition and no gingival erythema or bleeding secondary to tooth eruption. Exclusion criteria included infections, antibiotic or probiotic use within the prior 3 months, other autoimmune diseases, immunodeficiency, and malignancy.

Children in the JIA cohort satisfied the International League of Association for Rheumatology classification criteria for the diagnosis of oligoarticular, extended oligoarticular or polyarticular JIA [21], excluding enthesitis-related arthritis (ERA), psoriatic arthritis, undifferentiated arthritis, and systemic onset JIA. Subjects were enrolled in sequential order as they were seen in clinic, without selection by JIA subtype. Controls undergoing dental procedures for non-infectious causes (tooth removal for crowding, orthodontics or wisdom teeth removal) were recruited in the dental clinic. A second control group of healthy children was recruited from the community. The Seattle Children's Hospital Institutional Review Board (approval \#14045) reviewed all study activities. Informed parental permission, assent (for patients 10-17 years old), consent (for the one 18-year-old subject) and questionnaires were completed at the study visits. Smoking history was collected in sealed envelopes from subjects and parents separately.

\section{Clinical assessments}

Joint exams were performed for the JIA patients by pediatric rheumatologists. JIA disease activity (active vs. inactive) was determined by the ACR provisional criteria for clinical inactive disease (CID) [22]. Oral exams on all subjects were performed by dental providers. The gingival indices (GI), plaque indices (PI) [23], community periodontal indices (CPI) [24], and bleeding on probing (BOP) [25] were assessed with a standard periodontal probe of permanent teeth, including the four first permanent molars, upper right and lower left central incisors, in order to avoid scoring for sulci associated with eruption as periodontal pockets. For GI and PI, four surfaces (buccal, lingual, mesial and distal) were scored per tooth. GI and PI were calculated by dividing the number of gingival inflammation- or plaque-containing surfaces by the total number of scored surfaces. BOP was scored as presence or absence for each tooth examined and divided by the total number of teeth examined. Part way through the study, we learned that the dental group was advised not to brush teeth the morning of the study visit, as per the clinical protocol for patients undergoing general anesthesia. The GI is responsive to acute changes, such as poor hygiene for as little as $12 \mathrm{~h}$, but should not affect the BOP score. For this reason, the BOP was considered separately from the GI to dissect out the most specific sign of chronic gingival inflammation. The CPI was scored as the percentage of teeth examined. Decay missing filling teeth (DMFT) assessment was performed as a measurement of caries condition, as per the WHO Oral Health Survey Basic Methods [24]. Caries were scored by explorer or as clinically visible. Deciduous teeth, un-erupted teeth and teeth missing other than due to caries were not counted. If a tooth had both caries and a filling, it was marked as decay.

Patient/parent-reported health characteristics obtained by questionnaires included factors thought to play a role in oral health, (braces, gum bleeding, jaw pain, tooth pain, and mouth breathing). Smoking is a known risk factor for both periodontitis and rheumatoid arthritis [26, 27], and hence, patient and household cigarette smoking reports were solicited. 'Smoke currently' was defined as having smoked at least one cigarette in the last week or on at least one day in the last 30 days. Chronic smoking was defined as $>100$ cigarettes in a lifetime.

\section{Oral plaque collection and DNA extraction}

Plaque samples were collected by sterile endodontic paper points (Dentsply Sirona, York, PA) placed along 
the gingival margin of all 6 selected teeth on 22 patients with JIA (8 polyarticular, 10 oligoarticular, 4 extended oligoarticular) and 10 healthy controls. Because children have not developed periodontal pockets and thus lack accessible subgingival plaque, samples from the supragingival region were collected. Deoxyribonucleic acid (DNA) was extracted using the PowerSoil DNA Isolation Kit (MoBio, Carlsbad, CA) with a modified protocol: Prior to the addition of lysis buffer, Solution C1 (lysozyme) was added and incubated at $37^{\circ} \mathrm{C}$ for $30 \mathrm{~min}$, then $65^{\circ} \mathrm{C}$ and $95^{\circ} \mathrm{C}$ for $10 \mathrm{~min}$ each. For mechanical disruption, $0.5 \mathrm{~g}$ of $0.1 \mathrm{~mm}$ diameter Zirconia/Silica beads (BioSpec Products, Bartlesville, OK) were used. DNA was quantified using the Qubit 2.0 Fluorometer (Life Technologies, Carlsbad, CA).

\section{Library construction and $16 \mathrm{~s}$ rRNA sequencing}

Amplicon libraries of the 16S rRNA gene were generated [28]. Briefly, the V4 region was amplified with primers containing overhang sequence compatible with Illumina sequencing technology (Illumina, Inc., San Diego, CA). The amplicons were normalized using the SequalPrep Normalization Kit (Invitrogen), pooled in equal molar amounts, quantified using the Qubit 2.0 fluorometer (Life Technologies, Carlsbad, CA) and validated with the 2200 TapeStation (Agilent Technologies, Santa Clara, CA). The final amplicon pool was diluted, mixed with the Illumina-generated PhiX control library, and denatured according to standard Illumina protocol. Sequencing was performed on the Illumina MiSeq with the addition of custom primers as described [28] generating $2 \times 300 \mathrm{bp}$ paired-end reads. Sequence reads were trimmed to $250 \mathrm{bp}$ prior to analysis to remove the adapter sequence.

\section{Microbiome analysis}

Of the 32 samples sequenced, sequence output data was adequate for 27 . For core diversity analysis, only samples with at least 2700 sequence reads were used (random sampling rarified data to 2700 sequences each). The reads (4.14 million total) were quality filtered. Quantitative Insights Into Microbial Ecology (Qiime version 1.9.1) software was used for microbial analysis of the raw sequencing data [29]. Specific sequences that classified to the following organisms not known to be in the oral microbiota or common kit contaminants were removed: Enterobacteriaceae, Pseudomonadaceae, and Deinococcus [30]. The sequence length (151 bp) that remained after quality filtering allowed analysis of the microbiome data only to the genus level. Diversity analysis was performed based on operational taxonomic units (open references using 20 minimum sequences for an OTU and clustering using 97\% similarity in OTUs). The principal coordinate analysis plot (PCoA) was created using weighted unifrac distances between samples. The linear discriminant analysis effect size with the LEfse tool was created using normalized relative abundance to show differences in enriched microbes between the two groups [31]. Only microbial communities with a two log-fold difference or greater are shown in the LEfSe plot.

\section{Statistical analysis}

The statistical package $\mathrm{R}$ (version 3.3.2) was used for analysis of demographic and dental indices data. Demographic characteristics and patient reported health outcomes were compared between groups using analysis of variance (ANOVA) for quantitative variables and Fisher's exact test for categorical variables. Linear regression analysis was used to compare dental indices across groups with adjustment for demographic characteristics (age, gender, Caucasian race, household income and highest parent education). Formal comparisons of the mean values for dental indices were made between the JIA, dental and the healthy control groups. The analysis of similarities (ANOSIM) was used to test for statistical significance from the weighted unifrac distance matrix (PCoA plots). The ANOVA model was used to analyze the differences among bacteria genera between the polyarticular JIA and healthy control groups. $P$-values $<0.05$ were considered significant.

\section{Results}

\section{Patient characteristics}

A total of 158 sequential subjects were enrolled in the study: 85 patients with JIA, (22 oligoarticular, 17 extended oligoarticular and 46 polyarticular), and 62 dental patients. Eleven healthy controls were recruited from among the friends of JIA patients. Demographic and patient-reported health characteristics are reported in Table 1.

\section{Oral health}

Periodontitis is exceedingly rare in immunocompetent children, and we did not detect significant periodontal disease (with gingival sulcus depths $>3 \mathrm{~mm}$ ) in any subject. We did find that JIA patients tended to have higher periodontal indices, more similar to dental patients than to healthy controls. A CPI score $>0$ was present in $50.6 \%$ of JIA subjects, $55.4 \%$ of dental subjects and $36.4 \%$ of healthy controls (mean scores of $0.20,0.28$ and 0.12 , respectively). The differences between groups were not significant using logistic regression and a likelihood ratio test $(p=0.35)$.

We examined signs of gingival inflammation that may serve as early precursors to periodontitis. JIA patients had significantly higher BOP scores than dental patients (mean, (standard deviation, SD) 0.170 (0.248) vs. 0.076 $(0.208)$ ) after adjustment for demographic characteristics 
Table 1 Demographic data and patient-reported health characteristics

\begin{tabular}{|c|c|c|c|c|}
\hline & $\begin{array}{l}\text { Healthy Controls } \\
(N=11)\end{array}$ & $\begin{array}{l}\text { Dental Cases } \\
(N=62)\end{array}$ & $\begin{array}{l}\text { JlA Cases } \\
(N=85)\end{array}$ & $P$-Value \\
\hline${ }^{\mathrm{a} A g e}(\mathrm{yrs}$.$) , mean (SD)$ & $14.0(2.4)$ & $15.1(2.3)$ & $14.0(2.2)$ & 0.01 \\
\hline${ }^{\mathrm{a}}$ Female Gender, n (\%) & $2(18.2 \%)$ & $39(62.9 \%)$ & $69(81.2 \%)$ & $<0.001$ \\
\hline${ }^{a}$ Caucasian race, n (\%) & $11(100.0 \%)$ & $33(54.1 \%)$ & $65(78.3 \%)$ & 0.03 \\
\hline${ }^{a}$ Household income $>\$ 100,000, n(\%)$ & $9(81.8 \%)$ & $6(10.3 \%)$ & $24(30.8 \%)$ & $<0.001$ \\
\hline aparental Education >college, n (\%) & $8(72.7 \%)$ & $5(8.3 \%)$ & $18(22.2 \%)$ & $<0.001$ \\
\hline Braces, n (\%) & $1(9.1 \%)$ & $24(38.7 \%)$ & $20(23.8 \%)$ & 0.06 \\
\hline Gums bleed, n (\%) & $8(72.7 \%)$ & $26(41.9 \%)$ & $38(45.2 \%)$ & 0.19 \\
\hline Jaw pain, n (\%) & $2(18.2 \%)$ & $19(30.6 \%)$ & $33(39.3 \%)$ & 0.30 \\
\hline Tooth pain, n (\%) & $4(36.4 \%)$ & $22(35.5 \%)$ & $22(26.2 \%)$ & 0.41 \\
\hline Mouth breather, n (\%) & $2(18.2 \%)$ & $18(29.0 \%)$ & $29(34.5 \%)$ & 0.57 \\
\hline \multicolumn{5}{|l|}{ Patient smoking } \\
\hline${ }^{\mathrm{a}}$ Ever smoked, n (\%) & $0(0.0 \%)$ & $9(14.5 \%)$ & $0(0.0 \%)$ & $<0.01$ \\
\hline Smoke currently, n (\%) & $0(0.0 \%)$ & $4(6.5 \%)$ & $0(0.0 \%)$ & 0.06 \\
\hline${ }^{\mathrm{b}}$ Chronic smoker, n (\%) & $0(0.0 \%)$ & $2(3.2 \%)$ & $0(0.0 \%)$ & 0.29 \\
\hline \multicolumn{5}{|l|}{ Household smoking } \\
\hline${ }^{a}$ Smoke currently, n (\%) & $0(0.0 \%)$ & $20(32.2 \%)$ & $11(12.9 \%)$ & $<0.01$ \\
\hline${ }^{\mathrm{b}}$ Chronic smoker, n (\%) & $3(27.3 \%)$ & $29(46.8 \%)$ & $30(35.3 \%)$ & 0.27 \\
\hline
\end{tabular}

JIA Juvenile Idiopathic Arthritis

${ }^{a}$ Characteristics that were statistically different between groups $(P<0.05)$

${ }^{\mathrm{b}}$ Chronic smoking was defined as ever having smoked $\geq 100$ cigarettes

$(p=0.02$, Fig. 1a), suggesting that gingivitis is increased in JIA. In a linear regression analysis (Additional file 1 : Table S2), the adjusted difference between mean BOP scores in JIA patients versus dental patients was 0.104 with a standard error of $0.043(p=0.02)$. The JIA cohort trended toward higher mean BOP scores than the healthy control group, but this was not statistically significant $(p=0.41)$.

Additional aspects of oral health were assessed using specific dental indices (Fig. 1, Table 1, and Additional file 1: Table S1). We found that the JIA patients did not have overall worse oral hygiene, but rather better gingival indices compared to the dental group $(p=0.002)$ and non-significant trends toward better plaque and DMFT indices. Compared to healthy controls, the JIA group had significantly worse plaque indices $(p=0.049)$ and non-significant trends toward worse gingival and DMFT indices.

We investigated environmental factors that could influence outcomes. Within this study, the highest levels of education and income were noted in the healthy control group, followed by JIA and dental groups (Table 1). Covariate analysis within the dental cohort showed that the socioeconomic status (SES) influenced only the GI ( 0.81 in the lower income group vs. 0.37 in the higher income group, $p<0.05$ ).

Only nine subjects self-reported smoking, all in the dental cohort. In addition, dental controls had significantly higher exposure to current smokers in the household $(p<0.01)$, compared to the other two groups. We found that overall, patient smoking in the dental group was associated with DMFT $(p<0.01)$, but not gingivitis, plaque, or periodontitis. In the JIA patients with household member chronic smoking, higher DMFT scores were also found $(p<0.01)$. No associations were detected between household smoking status and other dental indices.

\section{JIA disease activity and oral health}

If gingival inflammation triggers systemic inflammation leading to synovitis, we might expect to detect a relationship between BOP and JIA disease activity. To test this hypothesis, we compared JIA patients with clinically inactive and active disease $(n=46,45)$ [22]. No association was found between JIA disease activity and any of the dental indices measured. In addition, there were no associations between dental index scores and subtypes of JIA (oligoarticular, polyarticular, extended oligoarticular) or disease duration (average of 5.5 years). Only four JIA patients, all with polyarticular JIA, had a history of anticitrullinated peptide antibodies (ACPA), and seven of rheumatoid factor. There was no increase in BOP in these patients compared to the other JIA patients.

We studied the effects of medications in the JIA cohort. In regression analysis we found no significant association between dental indices and either methotrexate $(n=38)$, biologics $(n=31)$, or corticosteroids $(n=6)$. 

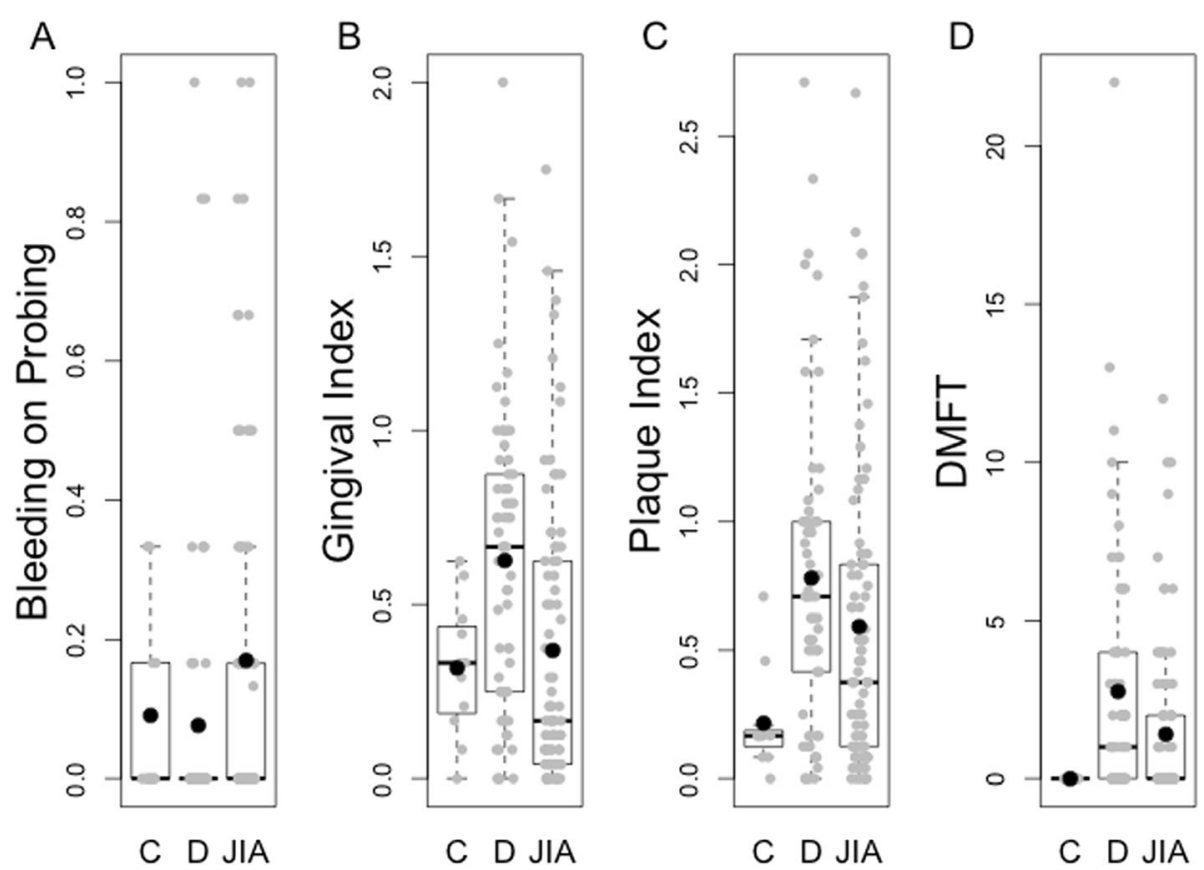

Fig. 1 Comparison of dental indices between groups. Comparison of dental indices between the Healthy Control (c), Dental (d) and Juvenile Idiopathic Arthritis (JIA) groups in Bleeding on Probing, Gingival Index, Plaque Index, and DMFT (Decay missing filling teeth) scores. Significant differences between JIA and Dental groups were found in BOP $(p=0.02)$ and GI scores $(p=0.002)$. Black bars, medians; filled circles, means

Non-steroidal anti-inflammatory drugs (NSAIDs) predispose to bleeding through altered platelet function, and thus could contribute to BOP. Although $16 \%$ of the JIA patients were taking NSAIDs, we detected no increase in $\mathrm{BOP}$ in this group.

\section{Plaque microbial communities}

We observed a trend toward higher plaque microbial diversity (using rarefraction measure of observed OTUs) in the JIA population compared to the healthy controls (Fig. 2a). However, the alpha diversity measure between the two groups calculated using the pairwise Wilcoxon test did not show statistical significance $(p=0.5)$. Since the use of only a single measure can result in different estimates of magnitude of change and subsequently different inferences, we adopted a varied diversity measure approach as commonly used in microbiome area of study to provide a more complete picture of the community structure. Further diversity analysis was conducted with six different measures, including the Chao1 index, abundance-based coverage estimator (ACE), Shannon index, Simpson index, inverse Simpson index, and, Fisher's alpha index. Using the Wilcoxon rank sum and pairwise Wilcoxon tests, no statistically significant differences were found.

Principal coordinate analysis showed visually evident clustering of JIA cohorts separate from healthy controls, but due to the small cohort and a few outliers, the difference was not statistically significant (Fig. 2b, ANOSIM $p=0.46$ ). A LEfSe plot comparing the whole JIA cohort vs. the healthy control group showed variation in the enriched microbes that have at least a two-log fold difference in linear discriminant analysis (LDA) score (Fig. 2c). Bacteria belonging to the genera Actinomyces and Corynebacterium were more abundant in the healthy control group, whereas, Streptococcus, Haemophilus, and Kingella were more prominent in the JIA group. Polyarticular JIA patients clinically resemble adult RA patients more closely than other types of JIA. To test for microbial profiles previously reported in adult RA [32], we specifically compared the polyarticular JIA and healthy control groups. Porphyromonas and Rothia were more abundant in the polyarticular JIA group, and Prevotella was higher in the healthy control group (Fig. 2d, $p<0.05$ ). A sub-analysis based on other JIA subtypes was also conducted. We found that the extended oligoarticular JIA group looked similar to healthy controls.

\section{Discussion}

Oral inflammation has been implicated as a trigger for adult inflammatory diseases, especially RA. Previous reports suggest that indicators of gingival inflammation may be elevated in JIA $[18,20]$. In this study, we found that JIA patients had increased BOP, a sign of gingival inflammation, despite otherwise superior oral health compared to children undergoing procedures in the dental clinic. BOP has been shown to correlate with risk for progression to periodontitis, size of inflammatory 


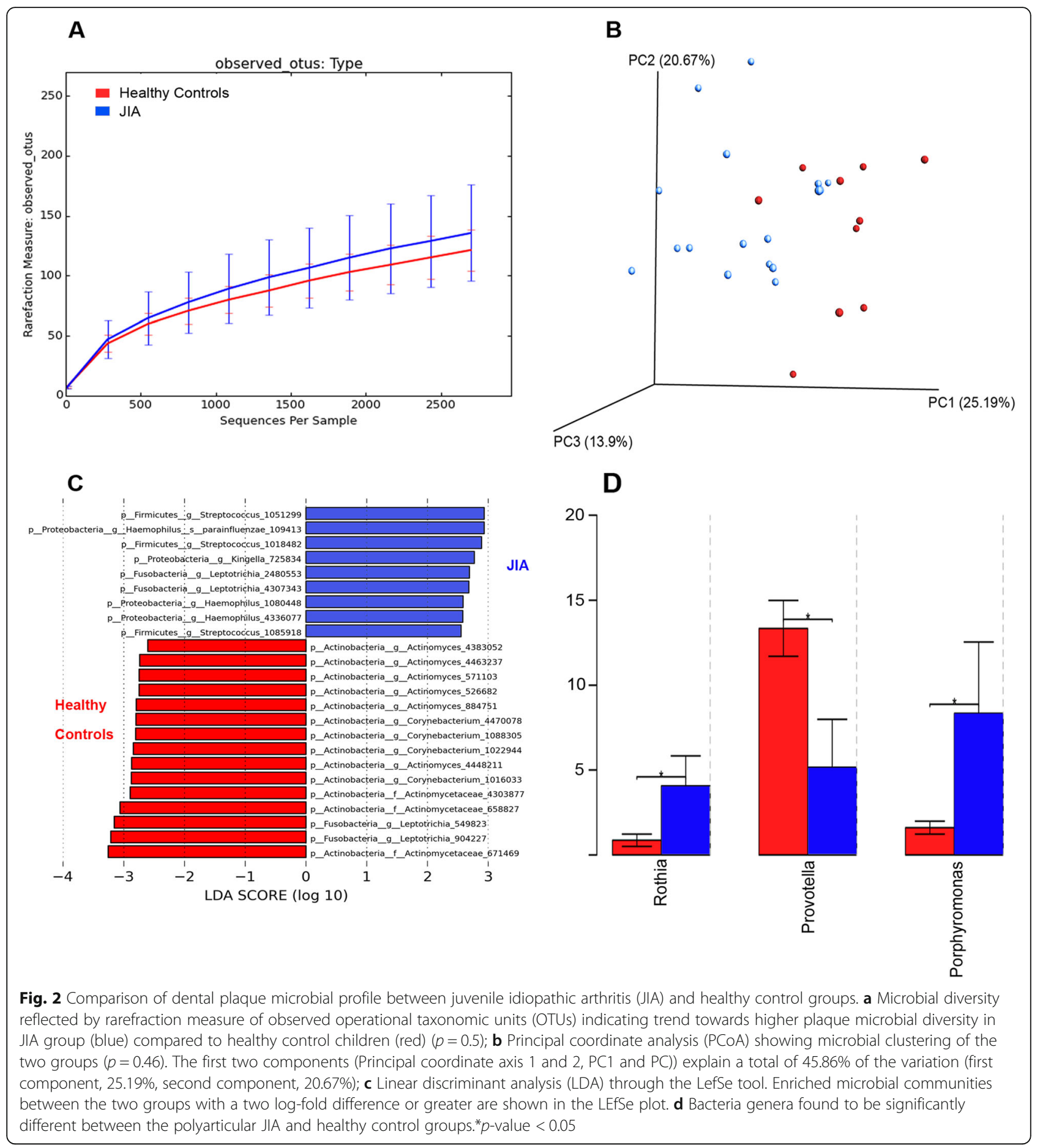

infiltrate in the gingival tissue, and levels of gingival proinflammatory mediators interleukins- $1 \beta$, interleukin-8, and matrix metalloproteinases [33-35], and thus is the most specific clinical indicator of gingival inflammation. Children from families with higher income and parental education tend to have better oral health-related quality of life [36], yet BOP scores were higher in the JIA cohort independent of SES. The reasons for increased gingivitis in JIA are not clear. NSAID and tumor necrosis factor inhibitors therapy have been used to treat periodontitis and gingivitis [37-39], and thus might decrease gingival or periodontal indices in this study. On the other hand, immunosuppression could theoretically allow for expansion of microbial communities normally checked by host immunity, similar to that seen in patients with primary immunodeficiency. Previous studies have suggested that 
JIA patients have poor oral health because of disability preventing adequate oral hygiene [16-18]. However, BOP scores in the JIA cohort were not affected by immunosuppression, NSAID use, JIA disease activity or JIA subtype, suggesting that there is another player, perhaps the microbiota, which could contribute to the pathogenesis of both JIA and gingivitis. Microbial communities can contribute to chronic local and systemic inflammation through altered mucosal immunity or protein citrullination $[5,6]$.

Comparison of oral microbial communities in the pediatric population with communities previously reported in RA is difficult, because the lack of deep sulci in children prevents collection of truly subgingival plaque. Periodontal pathogens are more commonly detected in deeper pockets [40]. Moreover, the analysis of the microbime in the current study was only specific to the genus level, precluding confident identification of known periopathogen species such as Porphyromonas gingivalis. While we did not find a predominance of known pathogens associated with severe periodontitis [41], mainly Prevotella intermedia, Fusobacterium nucleatum, Porphyromonas gingivalis, Tannerella forsythia and Treponema denticola in the combined JIA cohort, we did find bacteria belonging to the genus Porphyromonas in the polyarticular JIA group, similar to a previous report, in which a specific assay detected $P$. gingivalis in $21.5 \%$ of JIA patients [20]. Bacteria belonging to genera Haemophilus or Kingella, both gram-negative microbes, were elevated in the JIA cohort, interesting because of the known shift toward gram-negative anaerobes and spirochetes in periodontitis [15]. While not statistically significant, we failed to reject the null hypothesis that microbial diversity is similar in both groups. The JIA cohort tended towards a higher microbial diversity, similar to that previously found in gingiva with periodontitis or ulcerative gingivitis [42]. In new-onset RA, subgingival Prevotella and Leptotrichia were over-represented, whereas Corynebacterium and Streptococcus were underrepresented [4]. In contrast, we found Leptotrichia in both JIA and control groups, and Prevotella was lower in the polyarticular JIA group. Similar to adult RA, Corynebacterium was under-represented in JIA. It is important to recognize that the pathogenic processes at play in JIA are likely different than those in RA and the associations reported in RA may only be relevant for the subset of patients with seropositive JIA. In our study, only four patients had ACPAs and seven were RF positive, all of whom had polyarticular JIA.

Similarities exist between the pathogenesis of periodontal disease and JIA. Humoral immune responses to periodontal pathogens Porphyromonas gingivalis, Prevotella intermedia, and Aggregatibacter actinomycetemcomitans have been found in RA and JIA, and may contribute to pathogenesis [20, 43, 44]. Moreover, abundant evidence suggests that bidirectional crosstalk exists between microbial communities and the host immune response. It is also possible, therefore, that the association between oral inflammation secondary to specific microbiota and JIA are parallel processes with a common underlying immune defect, rather than a causal relationship. To this point, while induction of periodontitis can lead to arthritis in susceptible mice [45, 46], experimental arthritis can also trigger periodontitis [47]. Defective innate immune responses could play a role in proliferation of a specific microbiota that could in turn perpetuate the inflammatory responses. It is unclear whether JIA-associated immune dysfunction allows expansion of pathogenic bacteria that lead to activation of innate immunity.

The study has limitations that affected our ability to detect more notable associations of etiopathogenic importance. This is a cross-sectional study of a diverse population of treated patients, studied a few years after diagnosis of JIA. Further, the microbiota investigation was conducted on a smaller subsample of patients and thus had low power. The findings do not pertain to all JIA subtypes, as patients with ERA, psoriatic, systemic and undifferentiated categories were excluded. Unpredicted mismatches between JIA and control groups can influence oral health and microbial flora, including SES, race, smoking, and time since last brushing. The healthy control cohort had overall SES similar to the JIA cohort, but the sample size was small. No information was collected to estimate the frequency of dental care (i.e., time since last dental examination or cleaning, diet, mouthwash use). Because smoking has been established as a contributing factor to periodontitis and RA, [26, 27] we investigated the association of smoking with dental indices, but very few subjects reported smoking, making evaluation of the impact on arthritis risk or oral hygiene impossible. The study did examine for the first time the association of second-hand smoke with gingival inflammation in JIA, and no relationship was detected. TM) arthritis could contribute to poor oral hygiene because of difficulty in mouth opening, as suggested previously [18]. However only three patients had abnormal TMJ findings on clinical examination in the cohort reported here, and magnetic resonance imaging was not routinely conducted. Finally no corrections were made for multiple comparisons in this exploratory microbiome study, so suggested associations require confirmation in additional patient cohorts.

\section{Conclusions}

In this study, gingival inflammation and altered dental plaque microbial communities were associated with JIA despite overall normal oral health, and thus cannot be 
attributed to poor dental hygiene secondary to disability. The importance of monitoring and controlling oral health in children with JIA has yet to be determined through intervention trials. Understanding the role of oral pathogens in triggering and perpetuating chronic inflammation in JIA could lead to better approaches to prevention and treatment, but will require a better understanding of the immune responses to candidate oral microbes.

\section{Supplementary information}

Supplementary information accompanies this paper at https://doi.org/10. 1186/s12969-019-0387-5.

Additional file 1: Table S1. Mean and median data for Bleeding on Probing (BOP), Gingival Index (GI), Plaque Index (PI), and Decay, Missing, Filling Teeth (DMFT) scores between the three groups. The JIA group had lower $\mathrm{Gl}$ scores compared to the dental group $(p=0.002)$ and higher PI scores ( $p=0.049$ ) compared to healthy controls. No other differences were statistically significant. Table S2. Linear regression analysis to assess associations between oral health outcomes and patient group (JIA versus Dental), and demographic characteristics (regression coefficient estimate $\pm \mathrm{SE}, p$-value).

\section{Abbreviations}

ACE: Abundance-based Coverage Estimator; ANOSIM: Analysis of Similarities; ANOVA: Analysis of Variance; Anti-TNF: Anti-Tumor Necrosis Factor; BOP: Bleeding on Probing; CID: Clinically Inactive Disease; CPI: Community Periodontal Index; DMFT: Decay Missing Filling Teeth; DNA: Deoxyribonucleic Acid; ERA: Enthesitis Related Arthritis; Gl: Gingival Index; HLA: Human Leukocyte Antigen; JIA: Juvenile Idiopathic Arthritis; LDA: Linear Discriminant Analysis; LEfSe: Linear Discriminant Analysis Effect Size; NSAIDs: Non-steroidal Anti-Inflammatory Drugs; OTU: Operational Taxonomic Unit; PCOA: Principal Coordinate Analysis; PI: Plaque Index; RA: Rheumatoid Arthritis;

SES: Socioeconomic Status; TMJ: Temporomandibular Joint

\section{Acknowledgements}

Not applicable.

\section{Authors' contributions}

All authors read and approved the final manuscript. AS was senior author and SG was leading author. SG made a major contribution to study design, analysis and interpretation of data and writing the manuscript. PL made a major contribution to study design (dental component), analysis and interpretation of data and contributed to writing the manuscript. BL played a vital role in statistical analysis and interpretation of the data and contributed to writing the manuscript. SR made a major contribution to study design, data interpretation and revised the manuscript. JB, AK, EV, JK, CB, AR, GH and HL contributed to acquisition of data and reviewed the manuscript. RD helped with study design and interpretation of the oral health data. $\mathrm{KH}, \mathrm{MB}, \mathrm{HH}, \mathrm{SM}$ helped with processing plaque samples and analysis of microbiome data. JM played a major role microbial analysis and data interpretation. AS played a major role in study design, data analysis, data interpretation and manuscript writing.

\section{Funding}

Arthritis Foundation; Seattle Children's Foundation; NIH NIDCR R01DE023810 and R01DE020102; Pfizer Medical and Academic Partnership Program.

\section{Availability of data and materials}

The datasets used and/or analyzed during the current study are available from the corresponding author on reasonable request.

\section{Ethics approval and consent to participate}

Seattle Children's Hospital Institutional Review Board reviewed all study activities and approved the study (approval \#14045).

Informed parental permission, consent and assent (where applicable) were obtained from all participants.
Consent for publication

Not applicable.

\section{Competing interests}

The authors declare that they have no competing interest.

\section{Author details}

${ }^{1}$ Seattle Children's Hospital, 4800 Sand Point Way NE, Seattle, WA 98105, USA. ${ }^{2}$ Department of Pediatrics, University of Washington, 1959 NE Pacific St, Seattle, WA 98195, USA. ${ }^{3}$ Department of Periodontics, School of Dentistry, University of Washington, 1959 NE Pacific St, Seattle, WA 98195, USA. ${ }^{4}$ Center for Clinical and Translational Research, Seattle Children's Research Institute, 1900 9th Ave, Seattle, WA 98101, USA. ${ }^{5}$ Center for Immunity and Immunotherapies, Seattle Children's Research Institute, 1900 9th Ave, Seattle, WA 98101, USA. ${ }^{6}$ Department of Microbiology, University of Washington, 1959 NE Pacific St, Seattle, WA 98195, USA. ${ }^{7}$ Department of Genome Sciences, University of Washington, 1959 NE Pacific St, Seattle, WA 98195, USA. ${ }^{8}$ Department of Medicine, University of Washington, 1959 NE Pacific St, Seattle, WA 98195, USA.

Received: 29 July 2019 Accepted: 4 December 2019

Published online: 16 December 2019

\section{References}

1. Hersh AO, Prahalad S. Immunogenetics of juvenile idiopathic arthritis: a comprehensive review. J Autoimmun. 2015;64:113-24.

2. Vieira SM, Pagovich $O E$, Kriegel MA. Diet, microbiota and autoimmune diseases. Lupus. 2014;23(6):518-26.

3. Rosenstein ED, Greenwald RA, Kushner LJ, Weissmann G. Hypothesis: the humoral immune response to oral bacteria provides a stimulus for the development of rheumatoid arthritis. Inflammation. 2004;28(6):311-8.

4. Scher JU, Ubeda C, Equinda M, Khanin R, Buischi Y, Viale A, et al. Periodontal disease and the oral microbiota in new-onset rheumatoid arthritis. Arthritis Rheum. 2012;64(10):3083-94.

5. Lundberg K, Wegner N, Yucel-Lindberg T, Venables PJ. Periodontitis in RAthe citrullinated enolase connection. Nat Rev Rheumatol. 2010;6(12):727-30.

6. Araujo VM, Melo IM, Lima V. Relationship between periodontitis and rheumatoid arthritis: review of the literature. Mediat Inflamm. 2015;2015: 259074.

7. Stabholz A, Soskolne WA, Shapira L. Genetic and environmental risk factors for chronic periodontitis and aggressive periodontitis. Periodontol. 2010;53: 138-53.

8. Dosseva-Panova VT, Popova CL, Panov VE. Subgingival microbial profile and production of proinflammatory cytokines in chronic periodontitis. Folia Med (Plovdiv). 2014:56(3):152-60.

9. Al-Katma MK, Bissada NF, Bordeaux JM, Sue J, Askari AD. Control of periodontal infection reduces the severity of active rheumatoid arthritis. J Clin Rheumatol. 2007:13(3):134-7.

10. Ortiz P, Bissada NF, Palomo L, Han YW, Al-Zahrani MS, Panneerselvam A, et al. Periodontal therapy reduces the severity of active rheumatoid arthritis in patients treated with or without tumor necrosis factor inhibitors. J Periodontol. 2009:80(4):535-40.

11. Bello-Gualtero JM, Lafaurie GI, Hoyos LX, Castillo DM, De-Avila J, Munevar $J C$, et al. Periodontal disease in individuals with a genetic risk of developing arthritis and early rheumatoid arthritis: a cross-sectional study. J Periodontol. 2016;87(4):346-56

12. Barnes MG, Grom AA, Thompson SD, Griffin TA, Luyrink LK, Colbert RA, et al. Biologic similarities based on age at onset in oligoarticular and polyarticular subtypes of juvenile idiopathic arthritis. Arthritis Rheum. 2010;62(11):3249-58.

13. Hu Z, Jiang K, Frank MB, Chen $Y$, Jarvis JN. Complexity and specificity of the neutrophil Transcriptomes in juvenile idiopathic arthritis. Sci Rep. 2016:6:27453.

14. Pari A, llango $P$, Subbareddy $V$, Katamreddy V, Parthasarthy $H$. Gingival diseases in childhood - a review. J Clin Diagn Res. 2014;8(10):ZE01-4.

15. Mombelli A, Lang NP, Burgin WB, Gusberti FA. Microbial changes associated with the development of puberty gingivitis. J Periodontal Res. 1990;25(6): $331-8$.

16. Welbury RR, Thomason JM, Fitzgerald JL, Steen IN, Marshall NJ, Foster HE. Increased prevalence of dental caries and poor oral hygiene in juvenile idiopathic arthritis. Rheumatology (Oxford). 2003;42(12):1445-51. 
17. Ahmed N, Bloch-Zupan A, Murray KJ, Calvert M, Roberts GJ, Lucas VS. Oral health of children with juvenile idiopathic arthritis. J Rheumatol. 2004;31(8): 1639-43.

18. Leksell E, Ernberg M, Magnusson B, Hedenberg-Magnusson B. Intraoral condition in children with juvenile idiopathic arthritis compared to controls. Int J Paediatr Dent. 2008;18(6):423-33.

19. Pugliese C, van der Vinne RT, Campos LM, Guardieiro PR, Saviolli C, Bonfa E, et al. Juvenile idiopathic arthritis activity and function ability: deleterious effects in periodontal disease? Clin Rheumatol. 2016;35(1):81-91.

20. Romero-Sanchez C, Malagon C, Vargas C, Fernanda Torres M, Moreno LC, Rodriguez C, et al. Porphyromonas Gingivalis and lgG1 and IgG2 subclass antibodies in patients with juvenile idiopathic arthritis. J Dent Child (Chic). 2017:84(2):72-9.

21. Petty RE, Southwood TR, Manners P, Baum J, Glass DN, Goldenberg J, et al. International league of associations for Rheumatology classification of juvenile idiopathic arthritis: second revision, Edmonton, 2001. J Rheumatol. 2004;31(2):390-2.

22. Wallace CA, Ruperto N, Giannini E, Childhood A, Rheumatology Research A. Pediatric Rheumatology international trials $\mathrm{O}$, et al. preliminary criteria for clinical remission for select categories of juvenile idiopathic arthritis. J Rheumatol. 2004;31(11):2290-4

23. Loe $\mathrm{H}$. The gingival index, the plaque index and the retention index systems. J Periodontol. 1967;38(Suppl 6):610-6.

24. World Health Organization. Oral health surveys : basic methods. 4th ed. Geneva: World Health Organization; 1997. vii, 66 p. p

25. Newbrun E. Indices to measure gingival bleeding. J Periodontol. 1996;67(6): 555-61.

26. Tomar SL, Asma S. Smoking-attributable periodontitis in the United States: findings from NHANES III. National Health and nutrition examination survey. J Periodontol. 2000;71(5):743-51.

27. Stolt P, Bengtsson C, Nordmark B, Lindblad S, Lundberg I, Klareskog L, et al. Quantification of the influence of cigarette smoking on rheumatoid arthritis: results from a population based case-control study, using incident cases. Ann Rheum Dis. 2003;62(9):835-41.

28. Kozich JJ, Westcott SL, Baxter NT, Highlander SK, Schloss PD. Development of a dual-index sequencing strategy and curation pipeline for analyzing amplicon sequence data on the MiSeq Illumina sequencing platform. Appl Environ Microbiol. 2013;79(17):5112-20.

29. Caporaso JG, Kuczynski J, Stombaugh J, Bittinger K, Bushman FD, Costello EK, et al. QIIME allows analysis of high-throughput community sequencing data. Nat Methods. 2010;7(5):335-6.

30. Salter SJ, Cox MJ, Turek EM, Calus ST, Cookson WO, Moffatt MF, et al. Reagent and laboratory contamination can critically impact sequence-based microbiome analyses. BMC Biol. 2014;12:87.

31. Segata N, Izard J, Waldron L, Gevers D, Miropolsky L, Garrett WS, et al. Metagenomic biomarker discovery and explanation. Genome Biol. 2011; 12(6):R60.

32. Bender $P$, Burgin WB, Sculean A, Eick S. Serum antibody levels against Porphyromonas gingivalis in patients with and without rheumatoid arthritis - a systematic review and meta-analysis. Clin Oral Investig. 2017;21(1):33-42.

33. Tanner AC, Kent R Jr, Kanasi E, Lu SC, Paster BJ, Sonis ST, et al. Clinical characteristics and microbiota of progressing slight chronic periodontitis in adults. J Clin Periodontol. 2007;34(11):917-30.

34. Greenstein G, Caton J, Polson AM. Histologic characteristics associated with bleeding after probing and visual signs of inflammation. J Periodontol. 1981;52(8):420-5.

35. Teles R, Sakellari D, Teles F, Konstantinidis A, Kent R, Socransky S, et al. Relationships among gingival crevicular fluid biomarkers, clinical parameters of periodontal disease, and the subgingival microbiota. J Periodontol. 2010; 81(1):89-98.

36. Kumar S, Kroon J, Lalloo R. A systematic review of the impact of parental socio-economic status and home environment characteristics on children's oral health related quality of life. Health Qual Life Outcomes. 2014;12:41.

37. Paquette DW, Williams RC. Modulation of host inflammatory mediators as a treatment strategy for periodontal diseases. Periodontol. 2000;24:239-52.

38. Kadkhoda Z, Amirzargar A, Esmaili Z, Vojdanian M, Akbari S. Effect of TNFalpha blockade in gingival Crevicular fluid on periodontal condition of patients with rheumatoid arthritis. Iran J Immunol. 2016;13(3):197-203.

39. Kalantzis A, Marshman Z, Falconer DT, Morgan PR, Odell EW. Oral effects of low-dose methotrexate treatment. Oral Surg Oral Med Oral Pathol Oral Radiol Endod. 2005;100(1):52-62.
40. Haffajee AD, Cugini MA, Tanner A, Pollack RP, Smith C, Kent RL Jr, et al. Subgingival microbiota in healthy, well-maintained elder and periodontitis subjects. J Clin Periodontol. 1998;25(5):346-53.

41. Socransky SS, Haffajee AD, Cugini MA, Smith C, Kent RL Jr. Microbial complexes in subgingival plaque. J Clin Periodontol. 1998;25(2):134-44.

42. Paster BJ, Boches SK, Galvin JL, Ericson RE, Lau CN, Levanos VA, et al. Bacterial diversity in human subgingival plaque. J Bacteriol. 2001;183(12): 3770-83.

43. Albandar JM, DeNardin AM, Adesanya MR, Diehl SR, Winn DM. Associations between serum antibody levels to periodontal pathogens and early-onset periodontitis. J Periodontol. 2001;72(11):1463-9.

44. Albandar JM, Rams TE. Risk factors for periodontitis in children and young persons. Periodontol. 2002;29:207-22.

45. de Aquino SG, Talbot J, Sonego F, Turato WM, Grespan R, Avila-Campos MJ, et al. The aggravation of arthritis by periodontitis is dependent of IL-17 receptor a activation. J Clin Periodontol. 2017:44:881-91.

46. Yamakawa M, Ouhara K, Kajiya M, Munenaga S, Kittaka M, Yamasaki S, et al. Porphyromonas gingivalis infection exacerbates the onset of rheumatoid arthritis in SKG mice. Clin Exp Immunol. 2016;186(2):177-89.

47. Queiroz-Junior CM, Madeira MF, Coelho FM, Costa W, Bessoni RL, Sousa LF, et al. Experimental arthritis triggers periodontal disease in mice: involvement of TNF-alpha and the oral microbiota. J Immunol. 2011;187(7): $3821-30$.

\section{Publisher's Note}

Springer Nature remains neutral with regard to jurisdictional claims in published maps and institutional affiliations.
Ready to submit your research? Choose BMC and benefit from:

- fast, convenient online submission

- thorough peer review by experienced researchers in your field

- rapid publication on acceptance

- support for research data, including large and complex data types

- gold Open Access which fosters wider collaboration and increased citations

- maximum visibility for your research: over $100 \mathrm{M}$ website views per year

At BMC, research is always in progress.

Learn more biomedcentral.com/submissions 